ARTICLE

DOI: $10.1038 / \mathrm{s} 41467-018-05531-8$

\title{
Dipolar cations confer defect tolerance in wide-bandgap metal halide perovskites
}

Hairen Tan ${ }^{1,2}$, Fanglin Che ${ }^{1}$, Mingyang Wei ${ }^{1}$, Yicheng Zhao ${ }^{1}$, Makhsud I. Saidaminov ${ }^{1}$, Petar Todorović1, Danny Broberg 3,4, Grant Walters', Furui Tan 1,5, Taotao Zhuang ${ }^{1}$, Bin Sun (1) 1, Zhiqin Liang ${ }^{1}$, Haifeng Yuan (1) 1,6, Eduard Fron ${ }^{6}$, Junghwan Kim ${ }^{1}$, Zhenyu Yang (1) ${ }^{1}$, Oleksandr Voznyy (1) ${ }^{1}$, Mark Asta ${ }^{3,4}$ \& Edward H. Sargent (1) ${ }^{1}$

Efficient wide-bandgap perovskite solar cells (PSCs) enable high-efficiency tandem photovoltaics when combined with crystalline silicon and other low-bandgap absorbers. However, wide-bandgap PSCs today exhibit performance far inferior to that of sub-1.6-eV bandgap PSCs due to their tendency to form a high density of deep traps. Here, we show that healing the deep traps in wide-bandgap perovskites-in effect, increasing the defect tolerance via cation engineering-enables further performance improvements in PSCs. We achieve a stabilized power conversion efficiency of $20.7 \%$ for $1.65-\mathrm{eV}$ bandgap PSCs by incorporating dipolar cations, with a high open-circuit voltage of $1.22 \mathrm{~V}$ and a fill factor exceeding $80 \%$. We also obtain a stabilized efficiency of $19.1 \%$ for $1.74-e V$ bandgap PSCs with a high open-circuit voltage of $1.25 \mathrm{~V}$. From density functional theory calculations, we find that the presence and reorientation of the dipolar cation in mixed cation-halide perovskites heals the defects that introduce deep trap states.

\footnotetext{
${ }^{1}$ Department of Electrical and Computer Engineering, University of Toronto, 35 St. George Street, Toronto, ON M5S 1A4, Canada. ${ }^{2}$ National Laboratory of Solid State Microstructures, Collaborative Innovation Centre of Advanced Microstructures, Jiangsu Key Laboratory of Artificial Functional Materials, College of Engineering and Applied Sciences, Nanjing University, 210093 Nanjing, Jiangsu, China. ${ }^{3}$ Department of Materials Science and Engineering, University of California, Berkeley, CA 94720, USA. ${ }^{4}$ Materials Sciences Division, Lawrence Berkeley National Laboratory, Berkeley, CA 94720, USA. ${ }^{5}$ Key Lab of Photovoltaic Materials, Department of Physics and Electronics, Henan University, 475004 Kaifeng, China. ${ }^{6}$ Department of Chemistry, KU Leuven, Celestijnenlaan 200 F, B-3001 Leuven, Belgium. These authors contributed equally: Hairen Tan, Fanglin Che, Mingyang Wei. Correspondence and requests for materials should be addressed to H.T. (email: hairentan@nju.edu.cn) or to E.H.S. (email: ted.sargent@utoronto.ca)
} 
W ide-bandgap perovskite solar cells (PSCs) enable efficient monolithic tandem devices with crystalline silicon $(\mathrm{c}-\mathrm{Si})$ and other leading low-bandgap materials because of their high open-circuit voltage $\left(V_{\mathrm{oc}}\right)$ and tunable bandgaps $\left(E_{\mathrm{g}}\right)^{1-7}$. As the front subcell, the wide-bandgap PSCs should simultaneously exhibit high $V_{\text {oc }}$ (low $V_{\text {oc }}$ deficit), high fill factor (FF), and sufficient photocurrent density to match the bottom junction.

Varying halide composition enables perovskites with wide bandgap of around $1.7 \mathrm{eV}^{8}$, optimal for tandem cells with c-Si and other absorbers having about $1.1 \mathrm{eV}$ bandgap ${ }^{9-11}$. Stable wide-bandgap perovskites have been achieved by using mixed cations of formamidinium (FA) and cesium (Cs) on the A-site of the perovskite structure ${ }^{12,13}$. Using a composition of $\mathrm{Cs}_{0.17} \mathrm{FA}_{0.83} \mathrm{~Pb}\left(\mathrm{I}_{0.6} \mathrm{Br}_{0.4}\right)_{3}$, Snaith et al. obtained a stabilized power conversion efficiency (PCE) of around $16 \%$ and a maximum $V_{\text {oc }}$ of $1.2 \mathrm{~V}$ in $1.74-\mathrm{eV}$ perovskite solar cells ${ }^{12}$. Performance improvements in wide-bandgap PSCs were made by enlarging the grain $\operatorname{siz}^{14}{ }^{14}$, reducing interfacial traps ${ }^{15}$, adding rubidium ${ }^{16}$, and improving surface treatments ${ }^{17}$. Despite these advances, wide-bandgap PSCs still exhibit modest PCEs, large $V_{\mathrm{oc}}$ deficits $\left(E_{\mathrm{g}}-V_{\mathrm{oc}}\right)$, and low $\mathrm{FF}$, leading to performance well inferior to that of sub- $1.6 \mathrm{eV}$ bandgap PSCs ${ }^{18-21}$.

The poor performance of wide-bandgap PSCs is limited by a high trap density in the polycrystalline perovskite absorber. We reasoned that healing the deep traps in perovskite absorber-in effect, increasing the defect tolerance ${ }^{22,23}-$ could enable further performance improvements in widebandgap PSCs.

Sub-1.6 eV bandgap PSC strategies indicate that A-site cation compositional engineering is crucial to achieve the bestperforming devices $18,20,24,25$. Specifically, the most efficient PSCs contain a small amount of methylammonium (MA) cation. The mixing of MA and FA has primarily been utilized to achieve enhanced structural stability in $\mathrm{FAPbI}_{3}$-based perovskites ${ }^{26}$.

The electronic role-if one exists-played by the MA cation in the mixed cation-lead halide perovskites that led to the best-performing PSCs has seen initial studies of considerable interest in $\mathrm{MAPbI}_{3}$ perovskites; this important topic is much less studied in mixed cation perovskites.

In single cation $\mathrm{MAPbX}_{3}(\mathrm{X}=\mathrm{Cl}, \mathrm{Br}$, or I) perovskites, the collective rotation of dipolar MA cations has been proposed to enhance charge transport due to the formation of large polarons or the spatial localization of carriers ${ }^{27-29}$. The liquidlike reorientation of MA cations has been found to protect hot carriers in $\mathrm{MAPbX}_{3}$ perovskites ${ }^{30,31}$. The dipolar disorder of MA cations has been found to contribute to high dielectric constants in $\mathrm{MAPbX}_{3}$ perovskites, which should assist in screening of charged states or carriers ${ }^{32}$. Recent computational work has also revealed that deep trap states in $\mathrm{MAPbI}_{3}$ perovskite can be healed by dynamic rotation of MA cations in response to point charges ${ }^{33}$.

Inspired by these studies, we examine the impact of the MA cation on the electronic properties of FA-Cs-based mixed cation-halide wide-bandgap perovskites. We fabricate widebandgap PSCs and significantly boost the photovoltaic performance by incorporating a small amount of MA additive. We achieve a stabilized PCE of $20.7 \%$ in $1.65-\mathrm{eV}$ bandgap PSCs, with a high $V_{\text {oc }}$ of $1.22 \mathrm{~V}$ and a FF exceeding $80 \%$. The performance improvement is also validated for 1.74-eV bandgap PSCs, giving a stabilized PCE of $19.1 \%$ together with a high $V_{\text {oc }}$ of $1.25 \mathrm{~V}$. Using computational studies, we see a role of the MA cation in mixed perovskites, wherein the presence and the reorientation of dipolar MA cation eliminates-or renders innocuously shallow-electronic states associated with key defects. These states, in the absence of MA, would lead to deep traps.

\section{Results}

Characterization of wide-bandgap perovskite films. We fabricated wide-bandgap perovskite thin films with and without (controls) the dipolar MA cation (see Methods). Both compositions- $\mathrm{Cs}_{0.2} \mathrm{FA}_{0.8} \mathrm{~Pb}\left(\mathrm{I}_{0.75} \mathrm{Br}_{0.25}\right)_{3}$ (denoted $\mathrm{CsFA}$ ) and $\mathrm{Cs}_{0.05} \mathrm{MA}_{0.15} \mathrm{FA}_{0.8} \mathrm{~Pb}\left(\mathrm{I}_{0.75} \mathrm{Br}_{0.25}\right)_{3}$ (denoted CsMAFA) - exhibit a sharp absorption edge $\left(E_{\text {g,abs }}\right)$ at 1.67 and $1.65 \mathrm{eV}$, respectively (Fig. 1a). The absorption onset, rather than Tauc plot bandgap, is used here due to excitonic contributions to the spectrum close to the bandgap ${ }^{8,15}$ (Supplementary Fig. 1 and Supplementary Table 1). The partial replacement of Cs with MA shifts the absorption onset to slightly longer wavelength. Nuclear magnetic resonance (NMR) spectroscopy results further confirm that the ratios of MA and FA organic cations in the precursor solutions and the CsMAFA perovskite films are consistent (Supplementary Figs. 2 and 3 and Supplementary Table 2). We chose to experiment with perovskites having bandgaps of $1.65-1.70 \mathrm{eV}$, since previous modeling indicates that such bandgaps are ideal for top cell when monolithically combined with a c-Si real cell under practical conditions $\mathrm{s}^{9,34}$.

$\mathrm{X}$-ray diffraction (XRD) studies of films reveal a single cubic phase without $\mathrm{PbI}_{2}$ and non-perovskite yellow phases in each case (Fig. 1b). The XRD peak positions of CsMAFA film shift to slightly lower diffraction angles because of the larger ionic size in the case of MA, compared to Cs. Adding MA cations does not measurably affect the surface morphology. Both CsFA and CsMAFA films, each having a thickness around $600 \mathrm{~nm}$, are smooth and dense without noticeable pinholes. The two sets of films exhibit comparable grain size and similar grain growth (Fig. 1c, d and Supplementary Fig. 4).

Photoinduced phase segregation is a critical concern for widebandgap perovskites, given its detrimental effect on the stability of device performance under operation ${ }^{12,35,36}$. We carried out femtosecond transient absorption (TA) spectroscopy to further check the single phase as indicated by XRD and to investigate the photostability of CsMAFA perovskite films. Each sample was subjected to illumination using a 400-nm pump at a fluence of 4 $\mu \mathrm{J} \mathrm{cm}^{-2}$ (average pump power density of $20 \mathrm{~mW} \mathrm{~cm}^{-2}$ ) for at least $30 \mathrm{~min}$ in ambient air during TA measurements. Both CsFA and CsMAFA perovskite films showed photobleaching peaks that remained consistent at 711 and $719 \mathrm{~nm}$, respectively (Fig. 1e, f). We also checked the TA spectra of CsMAFA perovskite film at several different excitation wavelengths (Supplementary Fig. 5) and found no shift in the bleaching peak. These results indicate that wide-bandgap perovskite with CsMAFA triple cations is single phase in composition and phase stable under light illumination.

Improved photovoltaic performance with dipolar cation. We fabricated planar PSCs using the device architecture of ITO/ $\mathrm{TiO}_{2}-\mathrm{Cl} /$ perovskite/Spiro-OMeTAD/Au (Supplementary Fig. 4). We deposited a thick perovskite layer (about $600 \mathrm{~nm}$ ) in order to ensure substantially complete light harvesting above the bandgap. Chlorine-capped $\mathrm{TiO}_{2}\left(\mathrm{TiO}_{2}-\mathrm{Cl}\right)$ nanocrystal films were used to form the electron selective layer for contact passivation ${ }^{18}$. Figure $2 \mathrm{a}$ presents the statistical photovoltaic performance of PSCs using CsFA and CsMAFA perovskites measured under reverse scans. The CsMAFA PSCs exhibit considerably better performance than CsFA devices for all metrics. The average $V_{\text {oc }}$ increases by $50 \mathrm{mV}$ from $1.16 \pm 0.02$ to $1.21 \pm$ $0.01 \mathrm{~V}$. The average FF increases from $76 \pm 2$ to $79 \pm 2 \%$. The average $J_{\mathrm{sc}}$ increases from $19.9 \pm 0.5$ to $21.0 \pm 0.4 \mathrm{~mA} \mathrm{~cm}^{-2}$, mainly due to the slightly lower bandgap of CsMAFA. Correspondingly, the average PCE increases from $17.5 \pm 0.8$ to $20.0 \pm 0.5 \%$. 


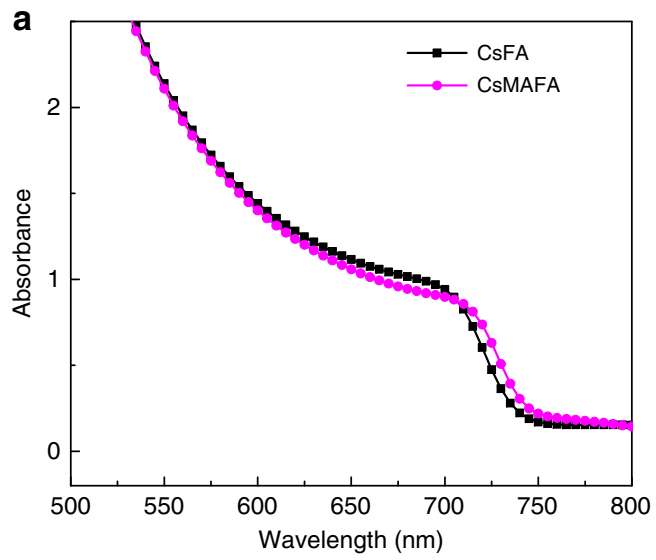

C

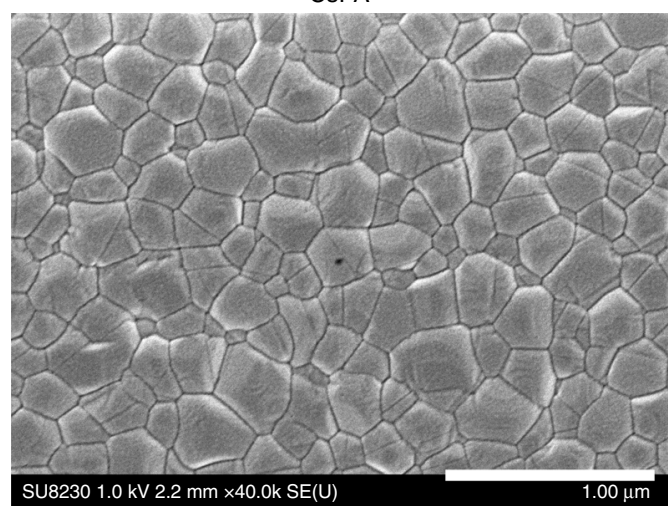

e

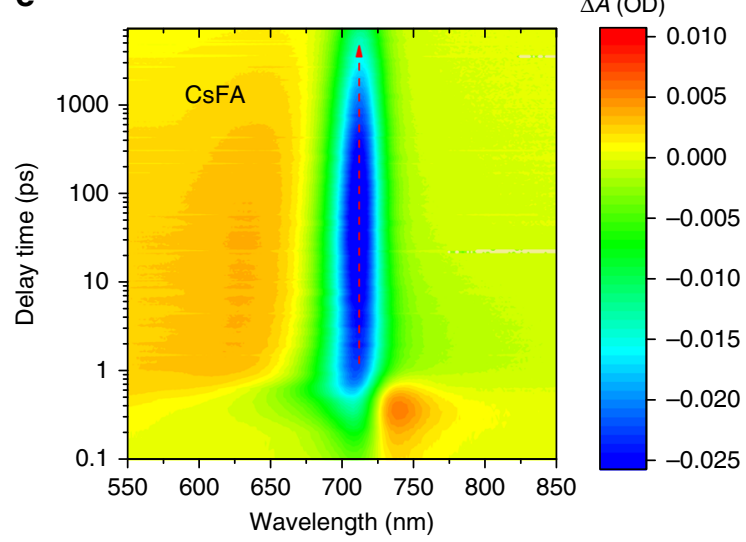

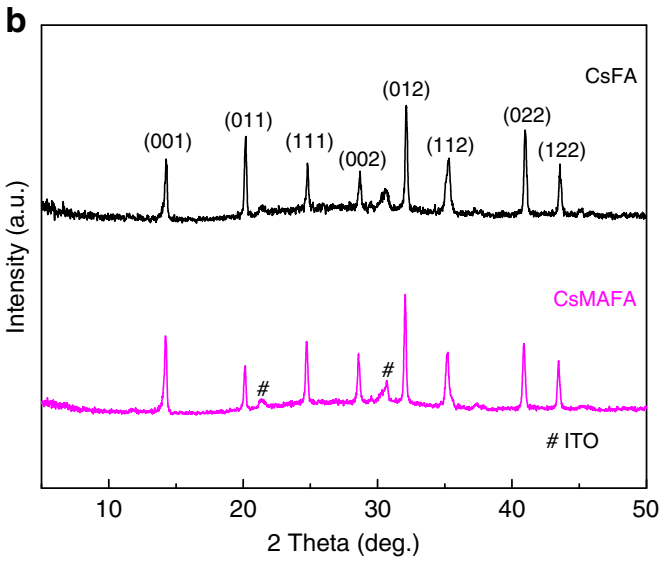

d

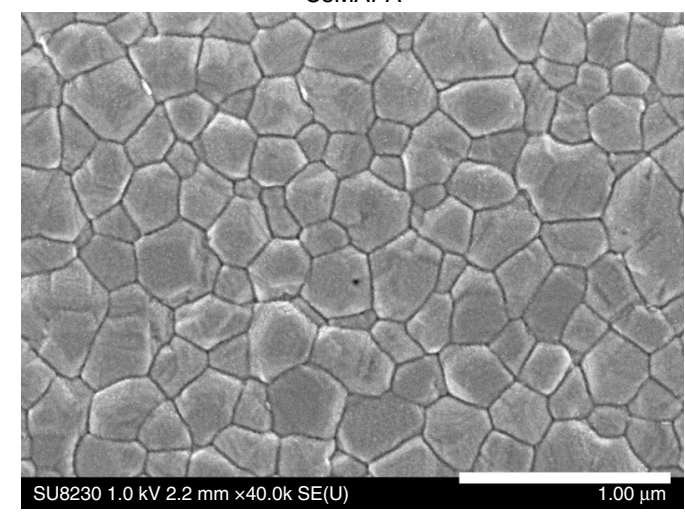

\section{f}

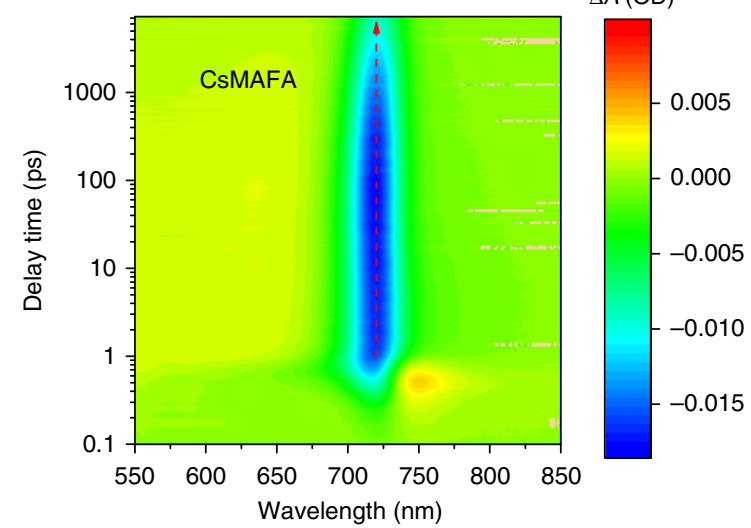

Fig. 1 Optical and structural characterization of wide-bandgap perovskite films. a Absorbance of perovskite films, corresponding to absorption onset of $1.67 \mathrm{eV}$ for CsFA and $1.65 \mathrm{eV}$ for CsMAFA. b XRD patterns of CsFA and CsMAFA perovskite films on ITO/TiO ${ }_{2}-\mathrm{Cl}$ substrates. c-d SEM images of CsFA and CsMAFA perovskite films on ITO/TiO $-\mathrm{Cl}$ substrates. The scale bars are $1 \mu \mathrm{m}$. e-f Transient absorption studies of CsFA and CsMAFA films on glass substrates. The vertical red dashed lines indicate the peak position of photobleaching band over a probe delay time of 1-7000 ps. OD optical density

The photovoltaic performances of the best-performing CsFA and CsMAFA perovskite solar cells are shown in Fig. $2 \mathrm{~b}$ and Table 1. The CsMAFA devices exhibit a best stabilized PCE of 20.7\% (Supplementary Fig. 6) and negligible hysteresis, whereas the CsFA devices have considerable hysteresis, in agreement with previous studies ${ }^{12,14,17}$. The integrated $J_{\text {sc }}$ values from EQE spectra (Fig. 2c) are consistent with the $J-V$ measurements (within 5\% deviations). The high FF values that exceed $80 \%$ and low $V_{\text {oc }}$ deficit in CsMAFA devices are comparable to those of the best-reported lower-bandgap (below $1.6 \mathrm{eV}$ ) $\mathrm{PSCs}^{18-20}$. The CsMAFA wide-bandgap PSCs achieved here exhibit a low $V_{\mathrm{oc}}$ deficit, sufficiently large $J_{\mathrm{sc}}$, and high FF and thus match well with high-efficiency c-Si solar cells ${ }^{37}$ needed in the future realization of monolithic tandem devices. To explore film uniformity, we fabricated large-area CsMAFA PSCs with an active area over 1 $\mathrm{cm}^{2}$ (Fig. 2d). The devices exhibit a maximum PCE of $19.3 \%$ with a low $V_{\text {oc }}$ deficit $\left(E_{\mathrm{g}}-V_{\mathrm{oc}}\right)$ of $410 \mathrm{mV}$. We tracked the operating stability of CsFA and CsMAFA perovskite solar cells at their maximum power point (MPP) conditions under one sun illumination (Supplementary Fig. 7). The CsMAFA solar cells have stable $V_{\text {oc }}$ under illumination, and they also exhibit comparable operating stability to the case of CsFA and maintain more than $97 \%$ of their initial efficiencies following continuous MPP operation for $11 \mathrm{~h}$. 

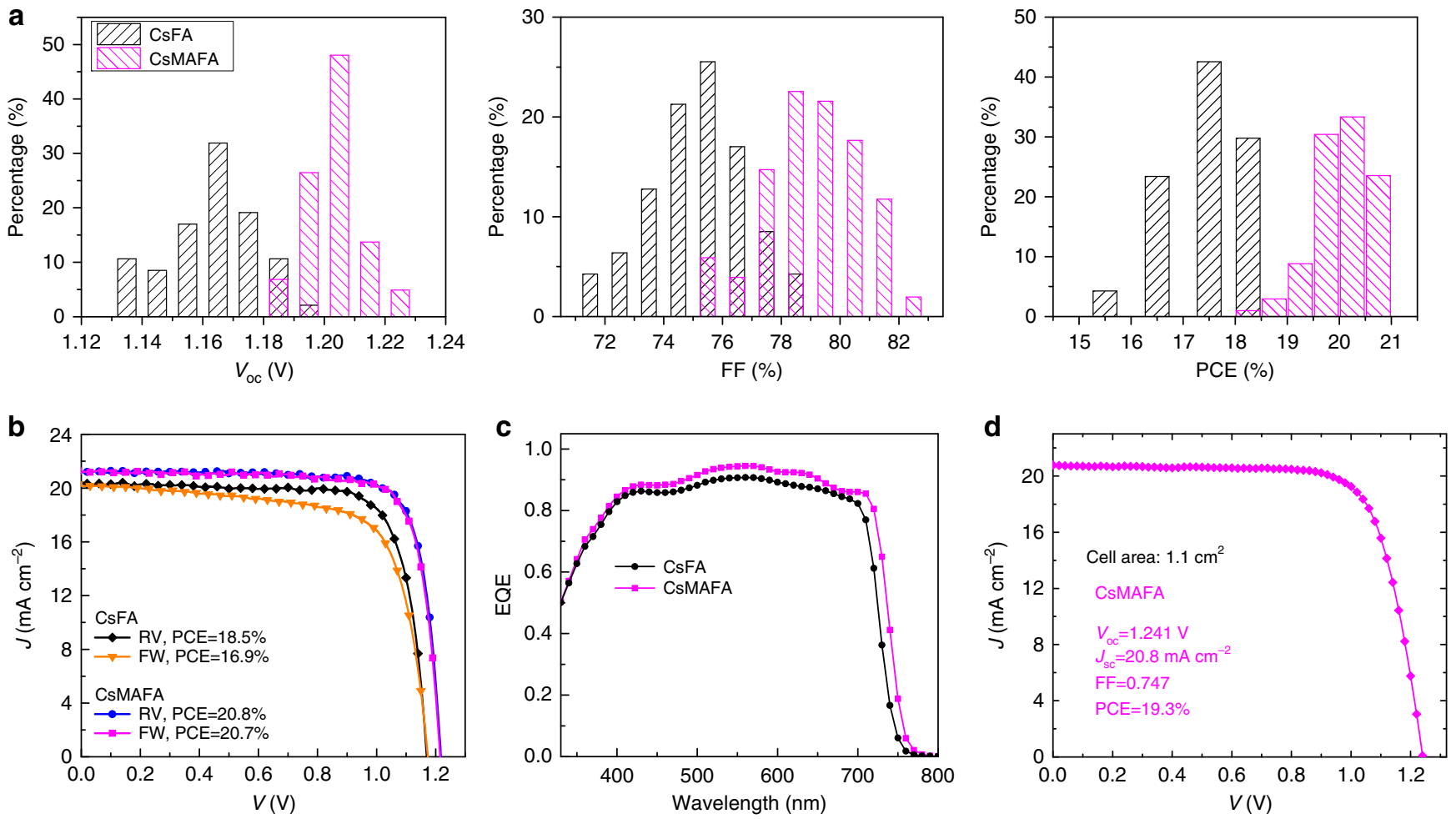

Fig. 2 Photovoltaic performance of wide-bandgap perovskite solar cells. a Histograms of $V_{\text {oc, }}$ FF, and PCE of 47 CsFA solar cells and 102 CsMAFA devices. b Reverse (RV) and forward (FW) J-V curves of best-performing CsFA and CsMAFA perovskite solar cells. c EQE curves of CsFA and CsMAFA perovskite solar cells, corresponding to integrated $J_{\mathrm{sc}}$ values of 19.4 and $20.7 \mathrm{~mA} \mathrm{~cm}^{-2}$, respectively. $\mathbf{d} J-V$ curve of best-performing CsMAFA solar cell with an active area of $1.1 \mathrm{~cm}^{2}$. CsFA and CsMAFA stand for the compositions of $\mathrm{Cs}_{0.2} \mathrm{FA}_{0.8} \mathrm{~Pb}\left(\mathrm{I}_{0.75} \mathrm{Br}_{0.25}\right)_{3}$ and $\mathrm{Cs}_{0.05} \mathrm{MA}_{0.15} \mathrm{FA}_{0.8} \mathrm{~Pb}\left(\mathrm{I}_{0.75} \mathrm{Br}_{0.25}\right)_{3}$, respectively

Table 1 Photovoltaic performance of best-performing wide-bandgap perovskite solar cells with and without MA cation

\begin{tabular}{|c|c|c|c|c|c|c|c|}
\hline Composition & $E_{g, a b s}(e V)$ & Scan direction & $V_{o c}(V)$ & $J_{s c}\left(m A c m^{-2}\right)$ & FF (\%) & PCE (\%) & Stabilized PCE (\%) \\
\hline \multirow{2}{*}{$\mathrm{Cs}_{0.2} \mathrm{FA}_{0.8} \mathrm{~Pb}\left(\mathrm{I}_{0.75} \mathrm{Br}_{0.25}\right)_{3}$} & 1.67 & RV & 1.17 & 20.4 & 77.3 & 18.5 & 17.6 \\
\hline & & FW & 1.17 & 20.2 & 71.3 & 16.9 & \\
\hline $\mathrm{Cs}_{0.05} \mathrm{MA}_{0.15} \mathrm{FA}_{0.8} \mathrm{~Pb}\left(\mathrm{I}_{0.75} \mathrm{Br}_{0.25}\right)_{3}$ & 1.65 & RV & 1.22 & 21.2 & 80.5 & 20.8 & 20.7 \\
\hline \multirow[t]{2}{*}{$\mathrm{Cs}_{0.17} \mathrm{FA}_{0.83} \mathrm{~Pb}\left(\mathrm{I}_{0.6} \mathrm{Br}_{0.4}\right)_{3}$} & 1.74 & RV & 1.22 & 18.7 & 75.6 & 17.2 & 16.7 \\
\hline & & FW & 1.21 & 18.4 & 70.5 & 15.7 & \\
\hline $\mathrm{Cs}_{0.12} \mathrm{MA}_{0.05} \mathrm{FA}_{0.83} \mathrm{~Pb}\left(\mathrm{I}_{0.6} \mathrm{Br}_{0.4}\right)_{3}$ & 1.74 & RV & 1.25 & 19.0 & 81.5 & 19.3 & 19.1 \\
\hline
\end{tabular}

Reduced recombination loss in MA-containing perovskites. We then turned to the study of the mechanistic origins of the performance improvement that arose upon the incorporation of MA into wide-bandgap PSCs. We used steady-state photoluminescence (PL), time-resolved PL (TR-PL), and impedance spectroscopy to study the charge recombination kinetics of perovskite films. The PL emission intensity of CsMAFA perovskite films is five times higher than that of CsFA samples (Fig. 3a). This indicates that adding MA cation reduces the non-radiative recombination sites in the perovskite film. To test the possibility that enhanced PL emission achieved by adding MA cations is mainly due to the change in film surface or grain boundaries, we spin-cast a thin layer of tri-n-octylphosphine oxide (TOPO) ligands on the as-obtained perovskite films with the goal of achieving surface/grain passivation, as demonstrated in previous reports $^{38,39}$. Both CsFA and CsMAFA perovskite films exhibited similar PL enhancements (Supplementary Fig. 8), indicating that the reduced non-radiative recombination in the CsMAFA perovskite films can be attributed principally to the improved bulk quality achieved by adding MA cations. We further carried out the TR-PL decays of perovskite films (Fig. 3b). The PL decay lifetime of CsMAFA film (516 ns) is longer than that of CsFA (326 ns), consistent with a reduced trap density in CsMAFA and suppressed non-radiative recombination channels.

We carried out thermal admittance spectroscopy (TAS) measurements to examine the trap density of states $\left(t_{\mathrm{DOS}}\right)$ in CsFA and CsMAFA perovskite solar cells. TAS probes shallow and deep trap states in solar cells ${ }^{40-42}$. The CsMAFA solar cells exhibited longer recombination lifetime than CsFA devices (Fig. 3c), implying slower charge recombination rate in CsMAFA. Figure $3 \mathrm{~d}$ presents the $t_{\mathrm{DOS}}$ of CsFA and CsMAFA perovskite solar cells measured under illumination at short-circuit condition. For CsFA devices, there are two obvious peaks: one, trap-state distribution with the peak position at $0.095 \mathrm{eV}$ and another, 

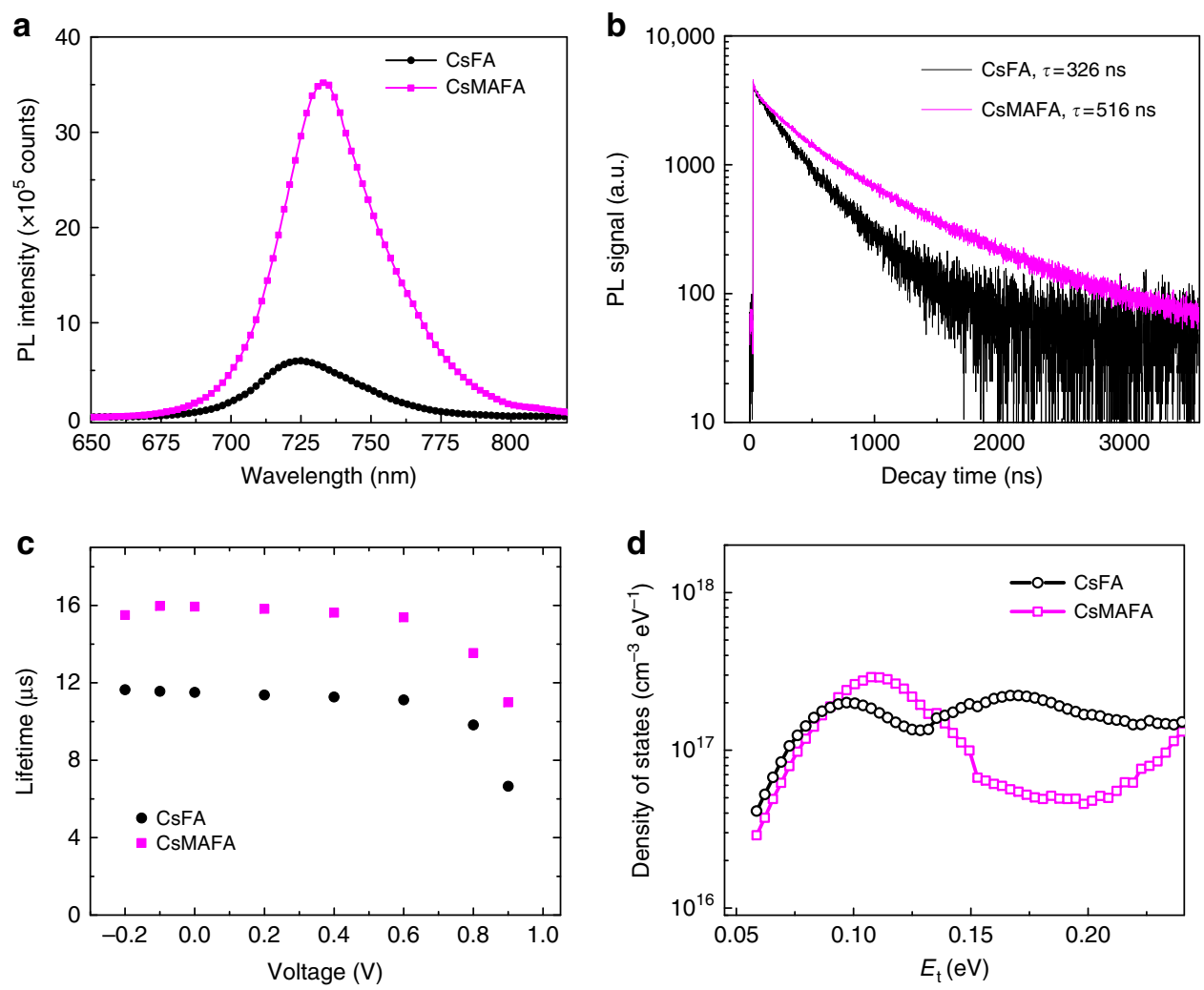

Fig. 3 Reduced trap density in MA-containing wide-bandgap perovskites. a Steady-state photoluminescence (PL) spectra and $\mathbf{b}$ time-resolved PL decay curves of CSFA and CSMAFA perovskite films deposited on glass substrates. c Recombination lifetimes of CsFA and CsMAFA solar cells extracted from impedance spectroscopy. d Trap density of states obtained from thermal admittance spectroscopy for CsFA and CsMAFA perovskite solar cells

deeper trap-state distribution with the peak position at $0.18 \mathrm{eV}$. The integrated trap densities of the shallow and deep trap levels are $0.6 \times 10^{16} \mathrm{~cm}^{-3}$ and $2.1 \times 10^{16} \mathrm{~cm}^{-3}$, respectively. For CsMAFA devices, however, there is only one shallow trap-state distribution with the peak position at $0.11 \mathrm{eV}$, corresponding to an integrated trap density of $1.1 \times 10^{16} \mathrm{~cm}^{-3}$.

Performance improvement in 1.74-eV bandgap solar cells. We further explored the impact of MA incorporation on the photovoltaic performance of other wide-bandgap PSCs. In ideal case (Shockley-Queisser limit for each junction), an optical bandgap of $1.75 \mathrm{eV}$ is desired for the top cell, in a tandem with c-Si. In this context, perovskite composition of $\mathrm{Cs}_{0.17} \mathrm{FA}_{0.83} \mathrm{~Pb}\left(\mathrm{I}_{0.6} \mathrm{Br}_{0.4}\right)_{3}$ has a near-ideal bandgap of $1.74 \mathrm{eV}^{12}$. By incorporating $5 \% \mathrm{MA}$ (a composition of $\mathrm{Cs}_{0.12} \mathrm{MA}_{0.05} \mathrm{FA}_{0.83} \mathrm{~Pb}$ $\left(\mathrm{I}_{0.6} \mathrm{Br}_{0.4}\right)_{3}$ ), we obtained perovskites having a substantially unaltered bandgap compared with $\mathrm{Cs}_{0.17} \mathrm{FA}_{0.83} \mathrm{~Pb}\left(\mathrm{I}_{0.6} \mathrm{Br}_{0.4}\right)_{3}$ (Supplementary Fig. 9). Stronger PL emission and longer PL decay lifetime were observed in the MA-containing perovskite films. Both $V_{\text {oc }}$ and FF of solar cells increased significantly after incorporation of MA (Table 1 and Supplementary Fig. 10a). $J_{\text {sc }}$ increases slightly as well, mainly due to enhanced charge collection in the long wavelength spectral region (Supplementary Fig. 10b). Consequently, stabilized PCE increases considerably from 16.7 to $19.1 \%$. The hysteretic behaviors in $J-V$ measurements are substantially eliminated upon MA incorporation. FF exceeds $80 \%$ and $V_{\mathrm{oc}}$ increases to $1.25 \mathrm{~V}$ after the incorporation of a small amount of MA. The $V_{\text {oc }}$ deficit $(490 \mathrm{mV})$ is higher than that of $1.65-\mathrm{eV}$ bandgap devices $(410 \mathrm{mV})$. Increasing the grain size and optimizing the interfaces may further reduce the $V_{\text {oc }}$ deficit $^{14,15}$
Defect healing by dipolar cations. We now explore how the incorporation of MA cation could reduce the trap-mediated recombination in mixed cation-halide perovskites. In $\mathrm{MAPbX}_{3}$ perovskites, the dynamic motion of MA cation was proposed to facilitate the formation of polarons ${ }^{28}$. Since the large effective mass of the polaron can shield the carriers from impurity scattering, polaron formation may reduce carrier scattering by defects or phonons and protect the hot carriers as well as bandedge carriers ${ }^{28-30}$. This has been offered as one important reason for the high-defect tolerance in perovskites. However, the formation of large polarons is predominantly associated with the deformation of the $\mathrm{PbX}_{3}{ }^{-}$framework (irrespective of the A-site cation type $)^{43,44}$. From transient absorption spectra, we found that, in the CsFA and CsMAFA systems, the films exhibit a comparable polaron formation time of about $0.4 \mathrm{ps}$, consistent with previously reported value (Supplementary Fig. 11a) ${ }^{43,44}$. We propose that the small MA additive concentration (5-15\%) used herein does not quantitatively impact the polaronic effect. We also carried out quasi-elastic neutron scattering (QENS) experiments to query the motion of cations in CsFA and CsMAFA perovskites (Supplementary Fig. 11b). The results indicate that CsFA and CsMAFA perovskites exhibit cation dynamic motion ${ }^{45-47}$.

One crucial difference between MA and FA/Cs cations is the much higher dipole moment for MA (about $2.3 \mathrm{D}$ ) compared with FA (about $0.2 \mathrm{D}$ ) and Cs (non-polar) due to their different molecular configuration (Supplementary Fig. 12) ${ }^{48}$. The liquid-like reorientation of dipolar MA cations has been found to protect hot carriers in $\mathrm{MAPbX}_{3}$ perovskites ${ }^{30,31}$. The dipolar disorder of MA cations has been found to contribute to high dielectric constants in $\mathrm{MAPbX}_{3}$ perovskites, which could assist in the screening of charged states or carriers (reducing the defect trapping cross-section) ${ }^{32}$. 

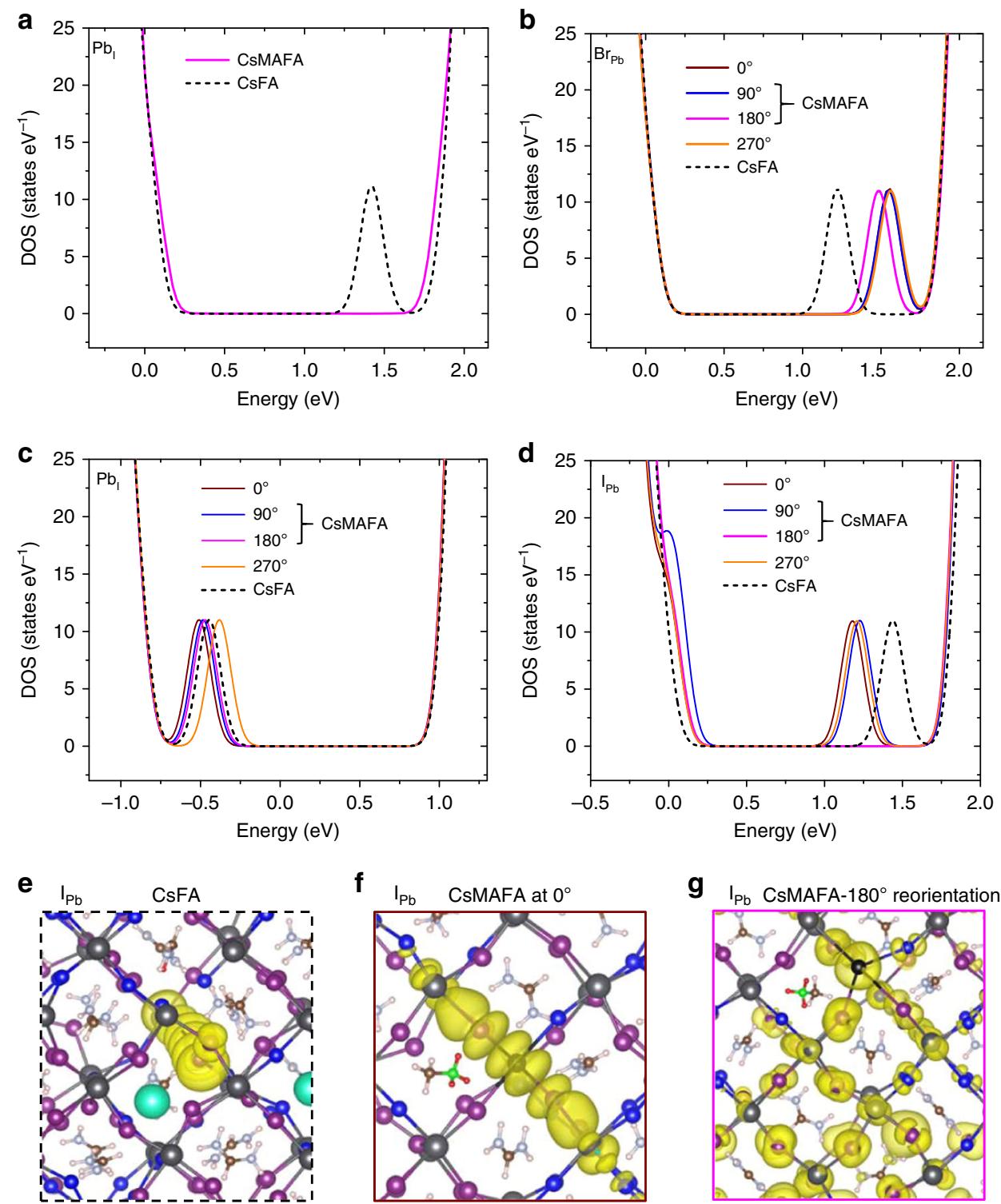

Fig. 4 Effects of MA reorientation on the electronic properties of charged defects. a Density of states (DOS) of CsFA and CsMAFA perovskites in the case of defect $\left(\mathrm{Pb}_{1}\right)$ that in-gaps states are present in CSFA perovskite, but not in CsMAFA perovskite, regardless of the reorientation direction. $\mathbf{b}-\mathbf{d}$ Density of states (DOS) of CsFA and CsMAFA perovskites in the cases of defects $\left(\mathrm{Br}_{\mathrm{Pb}}, \mathrm{Pb}_{\mathrm{i}}\right.$, and $\mathrm{I}_{\mathrm{Pb}}$ ) that introduce in-gap states in both perovskites, but the reorientation of MA cation renders shallower trap levels. e- $\mathbf{g}$ Wave functions of perovskites with the $I_{\mathrm{Pb}}$ defect: CsFA perovskite (e) and CsMAFA perovskite when the MA cation is randomized at $0^{\circ}(\mathbf{f})$ or is reoriented at the lowest energy direction $180^{\circ}(\mathbf{g})$

Recent computational work has also revealed that deep trap states in $\mathrm{MAPbI}_{3}$ perovskite can be healed by dynamic rotation of MA cations in response to point charges ${ }^{33}$.

While it is challenging to experimentally observe the reorientation of $\mathrm{MA}$ in response to defect sites and correlate the motion of MA cation with trap levels, we carried out density functional theory (DFT) calculations to explore how the MA cation influences the electronic properties of FA-Cs-based widebandgap perovskites (see Methods). We compared the materials (with composition close to our experimental materials) having vs. lacking the MA cation within these two formulae: $\mathrm{Cs}_{0.2} \mathrm{FA}_{0.8}$ $\mathrm{PbI}_{2} \mathrm{Br}$ (referred to as CsFA) and $\mathrm{Cs}_{0.05} \mathrm{MA}_{0.15} \mathrm{FA}_{0.8} \mathrm{PbI}_{2} \mathrm{Br}$ (referred to as CsMAFA) (see Supplementary Fig. 13).

We sought first to explore the impact of MA incorporation on the defect formation energies of the Schottky-type vacancy defects and charge-balanced antisite defects (Supplementary Fig. 14 and Supplementary Note 1). The partial incorporation of MA (15\%
MA) leads to slightly lower defect formation energies, except in the case of the $\mathrm{Pb}-\mathrm{I}$ antisite defect. We concluded that MA incorporation does not reduce the formation of defects.

We then proceeded to investigate how the presence and dynamic motion of dipolar MA cations close to the defect site affects the electronic properties of the defects that give rise to deep trap states in CsFA perovskite, antisite defects $\mathrm{Pb}_{\mathrm{I}}, \mathrm{Br}_{\mathrm{Pb}}$, and $\mathrm{I}_{\mathrm{Pb}}$, and interstitial defect $\mathrm{Pb}_{\mathrm{i}}{ }^{49-51}$. Here, we investigated reorientation of the dipolar $\mathrm{MA}$ cation. We investigated the effects that arise due to the fact that the dipole moment of MA is much stronger than that of FA, and that the kinetic barrier to the reorientation of $\mathrm{FA}$ is ten times higher than that of $\mathrm{MA}^{48}$. Compared to the MA cation, the FA cation has a negligible electrostatic effect in the presence of charges (free carriers or charged defects).

We investigated the first scenario, wherein the defect $\left(\mathrm{Pb}_{\mathrm{I}}\right)$ introduces in-gap trap states only in the CsFA perovskite (Fig. 4a). 
We found that, following MA incorporation, such in-gap states disappear in the CsMAFA perovskite, irrespective of the orientation direction of the MA cation.

In the second scenario, the defect $\left(\mathrm{Pb}_{\mathrm{i}}, \mathrm{Br}_{\mathrm{Pb}}\right.$, and $\left.\mathrm{I}_{\mathrm{Pb}}\right)$ introduces in-gap states in both CsFA and CsMAFA perovskites, but the reorientation of MA cation near the defect site renders shallower trap states (Fig. $4 \mathrm{~b}-\mathrm{d}$ ). For the $\mathrm{Br}_{\mathrm{Pb}}$ defect, $\mathrm{MA}$ incorporation with various MA reorientations consistently shifts the deep trap states to much shallower levels near the CBM (Fig. 4b). For the $\mathrm{Pb}_{\mathrm{i}}$ defect, with a certain $\mathrm{MA}$ rotation scenario (orange line in Fig. 4c), CsMAFA may have deeper traps than CsFA. As the MA cation rotates into more energetically favored orientations (Supplementary Table 3), the trap states shift to shallower levels than those in CsFA. In case of $\mathrm{I}_{\mathrm{Pb}}$ defect, it introduces deeper trap states in CsMAFA than CsFA when MA orientation is randomized (Fig. 4d). As the MA cation reorients to its lowest energy (most energetically favored) direction $\left(180^{\circ}\right.$, pink line in Fig. $4 \mathrm{~d}, \mathrm{NH}_{3}{ }^{+}$facing opposite to the defect), the deep trap states shift to very shallow levels near the VBM. The charge densities derived from the wave functions associated with the $\mathrm{I}_{\mathrm{Pb}}$ defect states in CsFA and CsMAFA perovskites are visualized in Fig. 4e-g. When the defect introduces deep trap states (Figs. 4e, f), the wave functions are localized near the charged defect site. After the MA reorients to its lowest energy direction, the trap states become very shallow near the VBM (Fig. 4d) and the wave function becomes delocalized (Fig. $4 \mathrm{~g}$ ), suggesting a reduced impact on carrier trapping.

\section{Discussion}

Our DFT studies reveal that the incorporation and reorientation of the dipolar MA cation in mixed perovskites offer the potential to heal the defects that introduce deep trap states in CsFA perovskite. The computational findings agree well with our experimental results, which show much reduced non-radiative recombination with MA incorporation. The trap-assisted Shockley-Read-Hall (SRH) recombination rate $R_{\mathrm{SRH}}$ can be expressed as Eq. (1) by assuming same capture cross-sections for electrons and holes ${ }^{52}$ :

$$
R_{\mathrm{SRH}}=V_{\mathrm{th}} \sigma N_{\mathrm{t}} \frac{n p-n_{\mathrm{i}}^{2}}{n+p+2 n_{\mathrm{i}} \cosh \left(\frac{E_{\mathrm{t}}-E_{\mathrm{i}}}{k_{\mathrm{B}} T}\right)}
$$

where $\sigma$ is capture cross-section of the traps, $n_{\mathrm{i}}$ is the intrinsic carrier concentration, $N_{\mathrm{t}}$ is trap density, $E_{\mathrm{t}}$ is trap energy level, $n$ is electron concentration, $p$ is hole concentration, $k_{\mathrm{B}}$ is Boltzmann constant, and $T$ is absolute temperature. The recombination rate $R_{\mathrm{SRH}}$ can be reduced in CsMAFA perovskite by decreasing the capture cross-section of traps due to the screening of defects by MA dipoles, or by reducing in-gap trap density or rendering shallower trap levels (schematically shown in Supplementary Fig. 15).

Charge carrier screening from (charged) defects enabled by $\mathrm{MA}$ is an overall consequence of the interactions between free carriers, charged defects, and dipoles. Charged defects generate a local electric field that attracts free carriers with opposite polarity and thus promotes trap-assisted SRH recombination. When a dipolar cation is in the proximity of a charged defect site, the dipolar cation reorients in response to the local electrical field due to the electrostatic interaction. This reduces the charge seen by free carriers and thus reduces the capture cross-section for nonradiative recombination. This screening effect depends on the direction of the dipole near the charged defect. As seen in our DFT studies, the in-gap trap states are closely related to the reorientation direction. When the local electric field $(\overrightarrow{\mathbf{F}})$ induced by charged defect is aligned with the dipole moment $(\overrightarrow{\mathbf{d}})$, the change in energy $U$ can be expressed as $(-\overrightarrow{\mathbf{d}} \cdot \overrightarrow{\mathbf{F}})$. We show that when the energy of hybrid halide perovskites is lowered by MA reorientation, the trap level becomes shallower (and the wave function becomes delocalized).

We investigated various defect types, including antisite, interstitial, and vacancy defects. We find that, for antisite and interstitial defects, the reorientation of the dipolar methylammonium (MA) cation in mixed perovskites heals the defects that introduce deep trap states (Fig. 4). We also investigated the vacancy defects that do not introduce in-gap trap states in CsFA and CsMAFA perovskites (Supplementary Fig. 16). Taking the I vacancy $\left(V_{\mathrm{I}}\right)$ as an example, we report that the reorientation of MA does not change the defect behavior in this case (Supplementary Fig. 17).

We note that our ground-state simulations are not without their limitations. As shown by Nan et al., trap-inefficient defects in the ground state (e.g., $\mathrm{Pb}$ and halide vacancies) may become trap-efficient defects in the excited state ${ }^{33}$. This means that those defects for which the orientation of MA is irrelevant in the ground state (e.g., $\mathrm{Pb}$ and I vacancies) may become deep traps following excitation. We expect that the dynamic motion of dipolar cation may also contribute to healing defects that only act as traps in the excited state. Mixed perovskites require a large number of atoms in DFT simulations, and our currently available computational capacity only allows us to carry out ground-state simulations. Further DFT studies (when larger computational capacity will be accessible) that take account of the excited-state conditions will enable further insights into dipolar cations and their role in defect healing in mixed cation-halide perovskites.

In summary, the incorporation of dipolar MA cation in photostable CsFA-based wide-bandgap perovskites significantly boosts solar cell efficiency by reducing the trap-assisted nonradiative recombination. The incorporation of $\mathrm{MA}$ does not necessarily reduce the formation of defects, but the incorporation of the dipolar MA cation in mixed cation-halide wide-bandgap perovskites heals deep trap defects, resulting in a more defecttolerant material. These findings shed light on defect healing in perovskite materials and pave the way to further increasing the efficiency of perovskite-enabled tandem photovoltaic devices.

\section{Methods \\ Materials. Unless stated otherwise, all materials were purchased from Sigma- Aldrich or Alfa-Aesar and used as received without further purification. The organic halide salts (MABr, FAI, and $\mathrm{FABr}$ ) were purchased from Dyesol Inc., Australia.}

Solar cell fabrication. The pre-patterned indium tin oxide (ITO, TFD Devices)coated glass was sequentially cleaned using acetone and isopropanol. A chlorinecapped $\mathrm{TiO}_{2}\left(\mathrm{TiO}_{2}-\mathrm{Cl}\right)$ nanocrystal electron transport layer (around $60 \mathrm{~nm}$ in thickness) was spin-coated twice on ITO substrate at $3000 \mathrm{rpm}$ for $30 \mathrm{~s}$ from the colloidal $\mathrm{TiO}_{2}-\mathrm{Cl}$ nanocrystal solution $\left(5 \mathrm{mg} \mathrm{mL}^{-1}\right)$. The $\mathrm{TiO}_{2}-\mathrm{Cl}$ solution was prepared according to previous work ${ }^{18}$. The $\mathrm{TiO}_{2}-\mathrm{Cl}$ film was then annealed on a hot plate at the displayed temperature of $150^{\circ} \mathrm{C}$ for $30 \mathrm{~min}$ in ambient air. After the substrates had cooled, we immediately transferred the substrates to a nitrogen-filled glovebox for the deposition of perovskite films. The perovskite precursor solutions (1.4 M) were prepared in a mixed solvent of DMF and DMSO (volume ratio 4:1) The molar ratios of $1 \mathrm{~mL}$ solution are listed as follows. $\mathrm{Cs}_{0.2} \mathrm{FA}_{0.8} \mathrm{~Pb}\left(\mathrm{I}_{0.75} \mathrm{Br}_{0.25}\right)_{3}$ : $0.07 \mathrm{mmol}$ CsI, $0.21 \mathrm{mmol} \mathrm{CsBr}, 1.12 \mathrm{mmol} \mathrm{FAI}, 0.98 \mathrm{mmol} \mathrm{PbI}_{2}$, and $0.42 \mathrm{mmo}$ $\mathrm{PbBr}_{2} ; \mathrm{Cs}_{0.05} \mathrm{MA}_{0.15} \mathrm{FA}_{0.8} \mathrm{~Pb}\left(\mathrm{I}_{0.75} \mathrm{Br}_{0.25}\right)_{3}: 0.07 \mathrm{mmol} \mathrm{CsI}, 0.21 \mathrm{mmol} \mathrm{MABr}, 1.12$ mmol FAI, $0.98 \mathrm{mmol} \mathrm{PbI}_{2}$, and $0.42 \mathrm{mmol} \mathrm{PbBr}_{2} ; \mathrm{Cs}_{0.17} \mathrm{FA}_{0.83} \mathrm{~Pb}\left(\mathrm{I}_{0.6} \mathrm{Br}_{0.4}\right)_{3}: 0.07$ mmol CsI, $0.168 \mathrm{mmol} \mathrm{CsBr}, 0.77 \mathrm{mmol} \mathrm{FAI}, 0.392 \mathrm{mmol} \mathrm{FABr}, 0.84 \mathrm{mmol} \mathrm{PbI}_{2}$, and $\left.0.56 \mathrm{mmol} \mathrm{PbBr}_{2} ; \mathrm{Cs}_{0.12} \mathrm{MA}_{0.05} \mathrm{FA}_{0.83} \mathrm{~Pb} \mathrm{I}_{0.6} \mathrm{Br}_{0.4}\right)_{3}: 0.07 \mathrm{mmol} \mathrm{CsI}, 0.098 \mathrm{mmol}$ $\mathrm{CsBr}, 0.07 \mathrm{mmol} \mathrm{MABr}, 0.77 \mathrm{mmol} \mathrm{FAI}, 0.392 \mathrm{mmol} \mathrm{FABr}, 0.84 \mathrm{mmol} \mathrm{PbI}_{2}$, and $0.56 \mathrm{mmol} \mathrm{PbBr}_{2}$. The perovskite films were deposited onto the $\mathrm{TiO}_{2}-\mathrm{Cl}$ substrates with a two-step spin-coating procedure. The first step was $2000 \mathrm{rpm}$ for $10 \mathrm{~s}$ with an acceleration of $200 \mathrm{rpm} \mathrm{s}^{-1}$. The second step was $6000 \mathrm{rpm}$ for $40 \mathrm{~s}$ with a ramp-up of $2000 \mathrm{rpm} \mathrm{s}^{-1}$. Chlorobenzene $(100 \mu \mathrm{L})$ was dropped on the spinning substrate during the second spin-coating step at $20 \mathrm{~s}$ before the end of the procedure. The substrate was then immediately transferred to a hot plate and heated at $100{ }^{\circ} \mathrm{C}$ for $30 \mathrm{~min}$. After cooling down to room temperature, the hole-transport layer was subsequently deposited on top of the perovskite film by spin coating at $3000 \mathrm{rpm}$ for $30 \mathrm{~s}$ using a chlorobenzene solution, which contained $72.3 \mathrm{mg} \mathrm{mL}^{-1}$ 
of Spiro-OMeTAD and $28.8 \mu \mathrm{L} \mathrm{mL}^{-1}$ of tert-butylpyridine, as well as $17.0 \mu \mathrm{L} \mathrm{mL}^{-1}$ of bis(trifluoromethane)sulfonimide lithium salt $\left(520 \mathrm{mg} \mathrm{mL}^{-1}\right.$ stock solution in acetonitrile). Finally, 100-nm Au contact was deposited on top of Spiro-OMeTAD by electron beam evaporation in an Angstrom Engineering deposition system.

Solar cell characterization. The current density-voltage $(J-V)$ characteristics were measured using a Keithley 2400 sourcemeter under the illumination of a solar simulator (Newport, Class A) at the light intensity of $100 \mathrm{~mW} \mathrm{~cm}^{-2}$, as checked with a calibrated reference solar cell (Newport). Unless otherwise stated, the $J-V$ curves were all measured in nitrogen atmosphere with a scanning rate of $50 \mathrm{mV} \mathrm{s}$ -1 (voltage step of $10 \mathrm{mV}$ and delay time of $200 \mathrm{~ms}$ ). Spectral mismatch factor of 1 was used. The steady-state PCE was measured by setting a bias voltage to $V_{\mathrm{MPP}}$ and then tracing the current density. $V_{\mathrm{MPP}}$ at maximum power point was determined from the reverse $J-V$ curve. The active area was determined by the aperture shade mask $\left(0.049 \mathrm{~cm}^{2}\right.$ for small-area devices and $1.1 \mathrm{~cm}^{2}$ for large-area devices) placed in front of the solar cell to avoid overestimation of the photocurrent density. EQE measurements were performed using Newport system (QuantX-300) with monochromatic light and white bias light (0.2 Sun). The system was calibrated by a certified silicon solar cell (Newport) each time before the EQE measurement.

\section{Femtosecond transient absorption measurements. Femtosecond laser pulses} were produced using a regeneratively amplified $\mathrm{Yb}: \mathrm{KGW}$ laser at a $5 \mathrm{kHz}$ repetition rate (Light Conversion, Pharos). The pump pulse was generated by passing a portion of the 1030-nm probe pulse through an optical parametric amplifier (Light Conversion, Orpheus) with the second harmonic of the signal pulse selected for $400 \mathrm{~nm}$ light. Both the pump and probe pulses (pulse duration $250 \mathrm{fs}$ ) were directed into an optical bench (Ultrafast, Helios), where a white light continuum was generated by focusing the $1030-\mathrm{nm}$ probe pulse through a sapphire crystal. The time delay was adjusted by optically delaying the probe pulse, with time steps increasing exponentially. A chopper was used to block every other pump pulse, and each probe pulse was measured by a charge-coupled device (CCD) after dispersion by a grating spectrograph (Ultrafast, Helios). Samples were prepared on a glass substrate and translated at $1 \mathrm{~mm} \mathrm{~s}^{-1}$ during measurement. Pump fluences were kept at $4 \mu \mathrm{J} \mathrm{cm}^{-2}$.

Steady-state PL and time-resolved PL measurements. Steady-state PL and timeresolved PL were measured using a Horiba Fluorolog time-correlated single-photon counting system with photomultiplier tube detectors. Light was illuminated from the top surface of the perovskite film. For steady-state PL measurements, the excitation source is a monochromated Xe lamp (peak wavelength at $520 \mathrm{~nm}$ with a line width of $2 \mathrm{~nm}$ ). For time-resolved PL, we used a green laser diode $(\lambda=540 \mathrm{~nm})$ as the excitation source, with an excitation power density of $5 \mathrm{~mW} \mathrm{~cm}^{-2}$. The PL decay curves were fitted with biexponential components to obtain a fast and a slow decay lifetime. The mean carrier lifetimes $\tau$ for the biexponential fit were calculated by the weighted average method.

Impedance spectroscopy. The impedance spectrum was measured using a potentiostat/galvanostat (AUT50690, PGSTAT204, the Netherlands) at different biases (from -0.20 to $1.05 \mathrm{~V}$ ). The frequency ranges from $1 \mathrm{MHz}$ to $0.01 \mathrm{~Hz}$ with 100 data points. The eigen-relaxation time or recombination time $\tau$ was fitted by using $R_{\mathrm{rec}} C_{\mathrm{rec}}$ (see equivalent circuit in Supplementary Fig. 18). Warburg impedance $\left(W_{\mathrm{s}}\right)$ is added to the equivalent circuit due to ion migration in the perovskite, which is manifested as a semi-infinite circle at the low-frequency part (below $10 \mathrm{~Hz}$ ). The density of the defect states was derived by the angular frequencydependent capacitance using the equation: $N_{\mathrm{t}}(f)=-\left(V_{\mathrm{bi}}-V_{\mathrm{app}}\right) / q W \mathrm{~K} T^{*}(\mathrm{~d} C / \mathrm{d} f)^{*} f$, where $V_{\mathrm{bi}}, W$, and $V_{\text {app }}$ stand for the build-in voltage, the width of the space charge region, and applied voltage, respectively. Then, the $x$-axis was turned from frequency to energetic distance by $E_{\mathrm{a}}=E_{\mathrm{t}}-E_{\mathrm{x}}$, where $E_{\mathrm{x}}$ is $E_{\mathrm{c}}$ or $E_{\mathrm{v}}$, using thermal admittance spectroscopy. Finally, we have the energetic defect distribution under different applied bias voltages as the equation: $N_{\mathrm{t}}\left(E_{\mathrm{a}}\right)=-\left(V_{\mathrm{bi}}-V_{\mathrm{app}}\right) / q W \mathrm{~K} T^{*}(\mathrm{~d} C /$ $\mathrm{d} f)^{\star} f$. For the identification of the energy levels of defects $\left(E_{\mathrm{a}}\right)$, we adopted the conversion parameter between the frequency and the energy level, considering the same device architecture and contacting layer ${ }^{53}$. At $T=300 \mathrm{~K}, E_{\mathrm{a}}=0.45-0.025 \mathrm{ln}$ $(2 \pi f)$ in $\mathrm{eV}$.

Additional characterization. High-resolution SEM images were obtained using the Hitachi SU8230 microscope with an accelerating voltage of $1 \mathrm{kV}$. A low accelerating voltage and a low beam current were deployed to reduce surface damage of perovskite films under electron beam bombardment. XRD patterns were collected using a Rigaku MiniFlex 600 diffractometer equipped with a NaI scintillation counter and monochromatized Copper Ka radiation $(\lambda=1.5406 \AA)$. Optical absorption measurements were carried out in a Lambda $950 \mathrm{UV} / \mathrm{Vis}$ spectrophotometer with an integration sphere. The ratio of MA and FA cations was quantified using ${ }^{1} \mathrm{H}$ nuclear magnetic resonance (NMR, Agilent DD2 500), in which $0.5 \mathrm{~mL}$ of solution was mixed with $0.1 \mathrm{~mL}$ of deuterated water $\left(\mathrm{D}_{2} \mathrm{O}\right)$ and $0.02 \mu \mathrm{L}$ of dimethyl sulfoxide (DMSO) was added as an internal standard. The onedimensional ${ }^{1} \mathrm{H}$ spectrum was measured with water suppression using a presaturation method. Quasi-elastic neutron scattering (QENS) measurements were done with the cold neutron chopper spectrometer (CNCS) at the Spallation Neutron Source (SNS) of the Oak Ridge National Laboratory. An incident neutron beam with an energy of $3.32 \mathrm{meV}$ was used. This energy yielded an elastic resolution of $102 \mu \mathrm{eV}$ and $Q$ measurement ranges of $0-2.5$. Perovskite single crystals were first ground into powders. The powders were then loaded under helium into cylindrical aluminum cans. Integration of spectra was for $|Q|=0.4-1.8$. An empty aluminum container was used for background subtraction.

Computational methods. We performed density functional theory (DFT) calculations using the Vienna Ab Initio Simulation Package (VASP) code ${ }^{54,55}$, wherein a planewave basis set was used under period boundary conditions. The exchangecorrelation functional that was employed was the Perdew-Burke-Ernzerhof (PBE) ${ }^{56}$ generalized-gradient approximation and the projector-augmented wave (PAW) method $^{57}$ for ion-electron interactions in the periodic system was used. The planewave kinetic energy cutoff was fixed at $400 \mathrm{eV}$, and the van der Waals interactions were modeled using DFT-D2 scheme of Grimme ${ }^{58}$. A supercell consisting of $108 \mathrm{ABX}_{3}$ units was employed in all calculations. Here, A can be a mixture of MA, $\mathrm{FA}$, and Cs, and X can be I or Br. For the CsFA configuration, the molar ratio of Cs: FA is 21:87 in order to be as close as possible to the experimental composition that gives a bandgap around $1.7 \mathrm{eV}$. To model the configuration of the CsMAFA perovskite, we partially replaced Cs with MA to have the molar ratio Cs: MA:FA $=5: 16: 87$ and kept the $\mathrm{Br}$ content the same as in the CsFA perovskite. For both CsFA and CsMAFA perovskites, the molar ratio of I:Br is 2:1. The calculated optimized geometries with disordered orientation of organic molecules (cations) for bulk CsFA and CsMAFA perovskites without defects are presented in Supplementary Fig. 13. The same setup was used for the bulk CsFA and CsMAFA perovskites with defects.

The Brillouin zone was sampled using a single (Gamma) k-point, and electronic convergence criterion of $10^{-7} \mathrm{eV}$ per formula unit was used. The computational cells were initially obtained using periodic repetition of unit cells and were then heated to $300 \mathrm{~K}$ using NVT molecular dynamics simulations to obtain random orientations of organic molecules. After 3-ps simulations using 1-fs time step, structures were cooled down to $0 \mathrm{~K}$ and were relaxed using a conjugate gradient algorithm until the energy converged within $10^{-5} \mathrm{eV}$ per formula unit. The reason that we did not apply the hybrid DFT functionals (i.e., HSE) or the spin orbital coupling (SOC) calculations is to closely relate to the experimental molar ratio of CsMAFA and CsFA perovskite materials; the system will run into memory issue (we were using BGQ-scinet supercomputer, which consist $1024 \mathrm{CPU}$ with 64 nodes per core) when running both HSE and SOC calculations. DFT-GGA calculations without SOC effects were shown to capture semi-quantitative behavior. Good agreement of the DFT bandgap between experiment and theory is largely attributed to large error cancelation ${ }^{49}$.

To examine the effects of organic cation orientation on the defect states of CsMAFA perovskites (as presented in Fig. 4), we fixed one single MA cation close to the defect site with certain orientation and relaxed the rest of the cell to obtain an optimized structure. Due to the large computational cells and low geometry, there is an extremely large number of possible ways to arrange MA cations. Thus, we only chose a single MA cation arrangement to draw our qualitative conclusions. We also note that in while calculating the density of states (as presented in Fig. 4), the Brillouin zone of the supercell was sampled by a finer Monkhorst-Pack mesh, corresponding to a grid of $2 \times 2 \times 1 \mathrm{k}$-points. The wave functions (partial charge density of electrons) of the trap states in CsFA and CsMAFA perovskites with defects were calculated by specifying the energy range of the bands using standard settings in the VASP code.

Data availability. All data that support the findings in this study are present in the paper and the Supplementary Information. Additional data related to this study are available from the corresponding authors on reasonable request.

\section{Received: 18 April 2018 Accepted: 8 July 2018}

Published online: 06 August 2018

\section{References}

1. Anaya, M., Lozano, G., Calvo, M. E. \& Míguez, H. ABX3 perovskites for tandem solar cells. Joule 1, 769-793 (2017).

2. Chen, B., Zheng, X., Bai, Y., Padture, N. P. \& Huang, J. Progress in tandem solar cells based on hybrid organic-inorganic perovskites. Adv. Energy Mater. 7, 1602400 (2017)

3. Hörantner, M. T. et al. The potential of multijunction perovskite solar cells. ACS Energy Lett. 2, 2506-2513 (2017).

4. Eperon, G. E., Hörantner, M. T. \& Snaith, H. J. Metal halide perovskite tandem and multiple-junction photovoltaics. Nat. Rev. Chem. 1, 0095 (2017).

5. Werner, J. et al. Efficient near-infrared-transparent perovskite solar cells enabling direct comparison of 4-terminal and monolithic perovskite/silicon tandem cells. ACS Energy Lett. 1, 474-480 (2016). 
6. Bush, K. A. et al. 23.6\%-efficient monolithic perovskite/silicon tandem solar cells with improved stability. Nat. Energy 2, 17009 (2017).

7. Eperon, G. E. et al. Perovskite-perovskite tandem photovoltaics with optimized band gaps. Science 354, 861-865 (2016).

8. Unger, E. L. et al. Roadmap and roadblocks for the band gap tunability of metal halide perovskites. J. Mater. Chem. A 5, 11401-11409 (2017)

9. Hörantner, M. T. \& Snaith, H. J. Predicting and optimising the energy yield of perovskite-on-silicon tandem solar cells under real world conditions. Energy Environ. Sci. 10, 1983-1993 (2017).

10. Jäger, K., Korte, L., Rech, B. \& Albrecht, S. Numerical optical optimization of monolithic planar perovskite-silicon tandem solar cells with regular and inverted device architectures. Opt. Express 25, A473 (2017).

11. Futscher, M. H. \& Ehrler, B. Modeling the performance limitations and prospects of perovskite/Si tandem solar cells under realistic operating conditions. ACS Energy Lett. 2, 2089-2095 (2017).

12. McMeekin, D. P. et al. A mixed-cation lead mixed-halide perovskite absorber for tandem solar cells. Science 351, 151-155 (2016).

13. Bush, K. A. et al. Compositional engineering for efficient wide band gap perovskites with improved stability to photoinduced phase segregation. ACS Energy Lett. 3, 428-435 (2018).

14. Yu, Y. et al. Synergistic effects of lead thiocyanate additive and solvent annealing on the performance of wide-bandgap perovskite solar cells. ACS Energy Lett. 2, 1177-1182 (2017).

15. Lin, Y. et al. Matching charge extraction contact for wide-bandgap perovskite solar cells. Adv. Mater. 29, 1700607 (2017).

16. Duong, T. et al. Rubidium multication perovskite with optimized bandgap for perovskite-silicon tandem with over 26\% efficiency. Adv. Energy Mater. 7, 1700228 (2017).

17. Zhou, Y. et al. Benzylamine-treated wide-bandgap perovskite with high thermal-photostability and photovoltaic performance. Adv. Energy Mater. 7, 1701048 (2017).

18. Tan, H. et al. Efficient and stable solution-processed planar perovskite solar cells via contact passivation. Science 355, 722-726 (2017).

19. Yang, W. S. et al. Iodide management in formamidinium-lead-halide-based perovskite layers for efficient solar cells. Science 356, 1376-1379 (2017)

20. Saliba, M. et al. Incorporation of rubidium cations into perovskite solar cells improves photovoltaic performance. Science 354, 206-209 (2016)

21. Jiang, Q. et al. Planar-structure perovskite solar cells with efficiency beyond 21\%. Adv. Mater. 29, 1703852 (2017).

22. Walsh, A. \& Zunger, A. Instilling defect tolerance in new compounds. Nat. Mater. 16, 964-967 (2017).

23. Meggiolaro, D. et al. Iodine chemistry determines the defect tolerance of leadhalide perovskites. Energy Environ. Sci. 11, 702-713 (2018).

24. $\mathrm{Hu}, \mathrm{Y}$. et al. Understanding the role of cesium and rubidium additives in perovskite solar cells: trap states, charge transport, and recombination. Adv Energy Mater. 1703057, 1703057 (2018).

25. Saliba, M. et al. Cesium-containing triple cation perovskite solar cells: improved stability, reproducibility and high efficiency. Energy Environ. Sci. 9, 1989-1997 (2016).

26. Jeon, N. J. et al. Compositional engineering of perovskite materials for highperformance solar cells. Nature 517, 476-480 (2015).

27. Ma, J. \& Wang, L. Nanoscale charge localization induced by random orientations of organic molecules in hybrid perovskite $\mathrm{CH} 3 \mathrm{NH} 3 \mathrm{PbI} 3$. Nano Lett. 15, 248-253 (2015).

28. Zhu, X.-Y. \& Podzorov, V. Charge carriers in hybrid organic-inorganic lead halide perovskites might be protected as large polarons. J. Phys. Chem. Lett. 6, 4758-4761 (2015).

29. Chen, Y. et al. Extended carrier lifetimes and diffusion in hybrid perovskites revealed by Hall effect and photoconductivity measurements. Nat. Commun. 7, 12253 (2016).

30. Zhu, H. et al. Screening in crystalline liquids protects energetic carriers in hybrid perovskites. Science 353, 1409-1413 (2016).

31. Guo, Z. et al. Long-range hot-carrier transport in hybrid perovskites visualized by ultrafast microscopy. Science 356, 59-62 (2017).

32. Anusca, I. et al. Dielectric response: answer to many questions in the methylammonium lead halide solar cell absorbers. Adv. Energy Mater. 7, 1700600 (2017).

33. Nan, G. et al. How methylammonium cations and chlorine dopants heal defects in lead iodide perovskites. Adv. Energy Mater. 8, 1702754 (2018).

34. Dupré, O., Niesen, B., De Wolf, S. \& Ballif, C. Field performance versus standard test condition efficiency of tandem solar cells and the singular case of perovskites/silicon devices. J. Phys. Chem. Lett. 9, 446-458 (2018).

35. Hoke, E. T. et al. Reversible photo-induced trap formation in mixed-halide hybrid perovskites for photovoltaics. Chem. Sci. 6, 613-617 (2015).

36. deQuilettes, D. W. et al. Photo-induced halide redistribution in organic-inorganic perovskite films. Nat. Commun. 7, 11683 (2016)

37. Battaglia, C., Cuevas, A. \& De Wolf, S. High-efficiency crystalline silicon solar cells: status and perspectives. Energy Environ. Sci. 9, 1552-1576 (2016)
38. deQuilettes, D. W. et al. Photoluminescence lifetimes exceeding $8 \mu$ s and quantum yields exceeding $30 \%$ in hybrid perovskite thin films by ligand passivation. ACS Energy Lett. 1, 438-444 (2016).

39. Braly, I. L. et al. Hybrid perovskite films approaching the radiative limit with over $90 \%$ photoluminescence quantum efficiency. Nat. Photonics 12, 355-361 (2018).

40. Walter, T., Herberholz, R., Müller, C. \& Schock, H. W. Determination of defect distributions from admittance measurements and application to $\mathrm{Cu}(\mathrm{In}$, Ga)Se $\mathrm{Se}_{2}$ based heterojunctions. J. Appl. Phys. 80, 4411-4420 (1996)

41. Shao, Y., Xiao, Z., Bi, C., Yuan, Y. \& Huang, J. Origin and elimination of photocurrent hysteresis by fullerene passivation in $\mathrm{CH}_{3} \mathrm{NH}_{3} \mathrm{PbI}_{3}$ planar heterojunction solar cells. Nat. Commun. 5, 5784 (2014).

42. Shao, Y., Yuan, Y. \& Huang, J. Correlation of energy disorder and open-circuit voltage in hybrid perovskite solar cells. Nat. Energy 1, 15001 (2016).

43. Miyata, K. et al. Large polarons in lead halide perovskites. Sci. Adv. 3, e1701217 (2017).

44. Bretschneider, S. A. et al. Quantifying polaron formation and charge carrier cooling in lead-iodide perovskites. Adv. Mater. 1707312, 1-8 (2018).

45. Leguy, A. M. A. et al. The dynamics of methylammonium ions in hybrid organic-inorganic perovskite solar cells. Nat. Commun. 6, 7124 (2015)

46. Li, B. et al. Polar rotor scattering as atomic-level origin of low mobility and thermal conductivity of perovskite $\mathrm{CH}_{3} \mathrm{NH}_{3} \mathrm{PbI}$. Nat. Commun. 8, 16086 (2017).

47. Fabini, D. H. et al. Universal dynamics of molecular reorientation in hybrid lead iodide perovskites. J. Am. Chem. Soc. 139, 16875-16884 (2017).

48. Frost, J. M. et al. Atomistic origins of high-performance in hybrid halide perovskite solar cells. Nano Lett. 14, 2584-2590 (2014).

49. Buin, A. et al. Materials processing routes to trap-free halide perovskites. Nano Lett. 14, 6281-6286 (2014).

50. Kang, J. \& Wang, L. W. High defect tolerance in lead halide perovskite CsPbBr ${ }_{3}$. J. Phys. Chem. Lett. 8, 489-493 (2017).

51. Ball, J. M. \& Petrozza, A. Defects in perovskite-halides and their effects in solar cells. Nat. Energy 1, 16149 (2016).

52. Shockley, W. \& Read, W. T. Statistics of the recombination of holes and electrons. Phys. Rev. 87, 835-842 (1952)

53. Duan, H.-S. et al. The identification and characterization of defect states in hybrid organic-inorganic perovskite photovoltaics. Phys. Chem. Chem. Phys. 17, 112-116 (2015)

54. Kresse, G. \& Hafner, J. Ab initio molecular dynamics for liquid metals. Phys Rev. B 47, 558-561 (1993).

55. Kresse, G. \& Furthmüller, J. Efficiency of ab-initio total energy calculations for metals and semiconductors using a plane-wave basis set. Comput. Mater. Sci. 6, 15-50 (1996)

56. Perdew, J. P., Burke, K. \& Ernzerhof, M. Generalized gradient approximation made simple. Phys. Rev. Lett. 77, 3865-3868 (1996).

57. Payne, M. C., Teter, M. P., Allan, D. C., Arias, T. A. \& Joannopoulos, J. D. Iterative minimization techniques for ab initio total-energy calculations: molecular dynamics and conjugate gradients. Rev. Mod. Phys. 64, 1045-1097 (1992).

58. Pack, J. D. \& Monkhorst, H. J. 'Special points for Brillouin-zone integrations'a reply. Phys. Rev. B 16, 1748-1749 (1977).

\section{Acknowledgements}

This publication is based in part on work supported by the US Office of Naval Research (Grant Award No.: N00014-17-1-2524), by an award (OSR-2017-CPF-3321-03) from the King Abdullah University of Science and Technology (KAUST), by the Ontario Research Fund Research Excellence Program, and by the Natural Sciences and Engineering Research Council (NSERC) of Canada. The work of H.T. was also supported by a Rubicon grant (680-50-1511) from the Netherlands Organization for Scientific Research (NWO) and by the Thousand Talent Program for Young Outstanding Scientists in China. D.B. and M.A. acknowledge the support by the U.S. Department of Energy, Office of Science, Office of Basic Energy Sciences, and Materials Sciences and Engineering Division, under Contract No. DE-AC02-05-CH11231. Materials Project program KC23MP. M.I.S. acknowledges the Government of Canada's Banting Postdoctoral Fellowship Program for financial support. The DFT simulation is supported by the IBM Canada Research and Development Center through the Southern Ontario Smart Computing Innovation Platform (SOSCIP). A portion of this research used resources at the Spallation Neutron Source, a DOE Office of Science User Facility operated by the Oak Ridge National Laboratory.

\section{Author contributions}

H.T. conceived the idea, designed the experiments, fabricated all the devices, and conducted the characterizations. F.C. carried out and interpreted the DFT studies. D.B., M.A and O.V. assisted the DFT studies. M.W. contributed to the PL/TA measurements with input from H.Y., E.F. and Y.Z. performed the impedance spectroscopy measurements. P. T. and Z.L. performed the XRD measurements. G.W. performed the neutron scattering measurements and M.I.S. grew the single crystals. F.T., T.Z., B.S., Z.Y. and J.K. assisted in 
material characterization. H.T. wrote the draft with inputs of M.W., F.C. and E.H.S improved the manuscript. All authors contributed to and commented on the paper.

\section{Additional information}

Supplementary Information accompanies this paper at https://doi.org/10.1038/s41467018-05531-8.

Competing interests: The authors declare no competing interests.

Reprints and permission information is available online at http://npg.nature.com/ reprintsandpermissions/

Publisher's note: Springer Nature remains neutral with regard to jurisdictional claims in published maps and institutional affiliations. (c) (i) Open Access This article is licensed under a Creative Commons Attribution 4.0 International License, which permits use, sharing, adaptation, distribution and reproduction in any medium or format, as long as you give appropriate credit to the original author(s) and the source, provide a link to the Creative Commons license, and indicate if changes were made. The images or other third party material in this article are included in the article's Creative Commons license, unless indicated otherwise in a credit line to the material. If material is not included in the article's Creative Commons license and your intended use is not permitted by statutory regulation or exceeds the permitted use, you will need to obtain permission directly from the copyright holder. To view a copy of this license, visit http://creativecommons.org/ licenses/by/4.0/.

(C) The Author(s) 2018 